Klimaschutz

\section{Die beste Schuldenbremse}

Die Corona-Krise führt in nie dagewesener Art und Weise zu volkswirtschaftlichen Herausforderungen. Kreditfinanzierte Konjunkturprogramme nicht nur in Deutschland und Europa, sondern in der ganzen Welt, stützen die Wirtschaft. Und das ist auch gut so, selten waren die Zinsen so niedrig. Ob Steuerstundung, Kurzarbeitergeld oder zinslose Darlehen - staatliche Garantien reduzieren langfristig ökonomische Risiken und eröffnen wirtschaftliche Chancen. Der Umbau der Wirtschaft hin zu Klimaschutz und Nachhaltigkeit muss ebenso nachhaltig vorangetrieben werden. Das jüngste Urteil des Bundesverfassungsgerichts mahnt politische Umsetzung an, die Lasten des Klimaschutzes nicht auf zukünftige Generationen zu verschieben. Zur Bewältigung der Klimakrise sind Investitionen und Staatshilfen für den notwendigen Umbau von Wirtschaft und Gesellschaft notwendig und sinnvoll.

Die Industrie ist für Deutschlands Volkswirtschaft elementar. Die deutsche Industrie muss zur Erreichung der Klimaziele in den kommenden zehn Jahren die Emissionen um etwa $24 \%$ im Vergleich zu heutigen Emissionen senken. Dies gelingt nur, wenn Produktionsprozesse möglichst rasch auf Klimaneutralität ausgerichtet werden. Beispielsweise muss die Fahrzeugfertigung den benötigten Stahl aus $\mathrm{CO}_{2}$-freien Verfahren gewinnen. Dies kann etwa durch eine Betriebsumstellung der Hochöfen von Kohle auf aus erneuerbaren Energien hergestellten Wasserstoff gelingen. Da in den kommenden Jahren ohnehin rund $50 \%$ aller Hochöfen altersbedingt ersetzt werden müssen, ist die Zeit günstig, diese jetzt zukunftsfähig und klimaschonend auszurichten. Grüner Wasserstoff ist ebenso für die Chemieindustrie oder aber als Antriebsstoff für Schwerlast-, Schiffs- und Flugverkehr in einer klimaneutralen Wirtschaft zentral. Auch die Automobilbranche muss die Produktionsprozesse umstellen und im Individualfahrzeugbereich verstärkt auf Elektromobilität setzen. Aber auch im Schwerlastbereich werden klimaschonende Antriebe benötigt. Umfangreiche Modernisierungen sind somit dringend erforderlich.

Seit Langem leidet die deutsche Wirtschaft, vor allem die Industrie, an Investitionsschwäche. Dieser Investitionsstau kann nun mit gezielten Allianzen durch Wirtschaftshilfen überwunden werden. Das Geld sollte gezielt für Klimaschutz bereitgestellt werden, etwa für Digitalisierung,

(C) Der/die Autor:in(nen) 2021. Open Access: Dieser Artikel wird unter der Creative Commons Namensnennung 4.0 International Lizenz veröffentlicht (creativecommons.org/licenses/by/4.0/deed.de).

Open Access wird durch die ZBW - Leibniz-Informationszentrum Wirtschaft gefördert.
Smart Grids, Ladeinfrastruktur, klimaschonende Treibstoffe, Batterie- und Solarzellenproduktion oder Wasserstoff für die Schwerindustrie. Auch der Schienenverkehr muss gestärkt werden. Nur dann wird der Umbau hin zu einer klimaneutralen Wirtschaft gelingen, wie wir es im Rahmen internationaler Vereinbarungen verbindlich festgelegt haben. Viele Firmen orientieren sich seit einiger Zeit um und investieren bereits in klimafreundliche Technologien, etwa bei der Umstellung der Stahlproduktion auf Wasserstoff oder klimaschonende Treibstoffe. Wenn die Politik rückwärts- statt zukunftsgewandt agiert, laufen die Unternehmen Gefahr, dass ihre Investitionen unrentabel werden und innen der weitere Umbau durch solche politischen Verzögerungen nicht gelingt. Diese Unternehmen brauchen Planungssicherheit und langfristig verlässliche Rahmenbedingungen. Dazu gehört die Einhaltung der Klimabeschlüsse.

Dekarbonisierung, Digitalisierung, Dezentralisierung und Demokratisierung sind die Schlüsselbegriffe der Zukunft. Vor allem die Digitalisierung wird auch in der Energiewirtschaft nicht mehr wegzudenken sein. Wir brauchen dringender denn je dezentrale Netze, welche digital verknüpft werden zu virtuellen Kraftwerken. Es ist technisch kein Problem, dass Häuser mehr Energie erzeugen, als sie selbst verbrauchen. Sie sind Kleinkraftwerke, die wir zusammenschalten können. Dies ist eine Riesenchance für die digitale Energiewende. Wir dürfen die derzeitige Krise nicht ungenutzt lassen.

Die deutsche Wirtschaft wird sich nur dann schnell erholen und zugleich die wichtige strukturelle Transformation zur Erreichung der Klimaziele bewerkstelligen, wenn die privaten Investitionen in den kommenden Monaten stabilisiert werden können. Zudem müssen die öffentlichen und kommunalen Investitionen, vor allem zur Verbesserung der Infrastruktur, gestärkt werden. Ein Investitionsprogramm stärkt die Wachstumspotenziale und Resilienz der deutschen Wirtschaft. Dies erleichtert auch die zukünftige Rückzahlung der aufgenommenen Schulden. Zudem stärkt ein Investitionsprogramm die gesamtwirtschaftliche Nachfrage. Außerdem bietet ein zielgerichtetes wirtschaftliches Erholungsprogramm die Chance, die deutsche Wirtschaft durch Investitionen konsequent nachhaltig auszurichten und die notwendige sozialökologische Transformation zu unterstützen. Der Klimawandel kostet, der Klimaschutz nützt der Wirtschaft, der Gesellschaft und den zukünftigen Generationen. Klimaschutz ist die beste Schuldenbremse für die Industrie und Wirtschaft insgesamt.

Claudia Kemfert

DIW und Leuphana Universität sekretariat-evu@diw.de 